\title{
Conductivity in Ferroelectric Barium Titanate: Electrons versus Oxygen Vacancies
}

\author{
M. Tyunina
}

\begin{abstract}
Mobile oxygen vacancies are increasingly wider believed to be responsible for electrical conductivity in perovskite oxide ferroelectrics. Here, this hypothesis is debated. The smallsignal conductivity is investigated in oxygen-deficient films of barium titanate, where oxygen vacancies are epitaxially clamped and immobile. The observed behavior of conductivity as a function of temperature and frequency evidences pure electronic processes. Importantly, it is shown that these processes mimic motion of oxygen vacancies, which are immobile. It is also demonstrated that under applied dc electric field, the electronic processes lead to such effects as coloration and degradation, which before were plausibly ascribed to migration of oxygen vacancies. Finally, it is concluded that the hypothesis of mobile oxygen vacancies is misleading.
\end{abstract}

Index Terms - Ferroelectric devices, conductivity.

\section{INTRODUCTION}

$\mathrm{E}$ LECTRICAL conductivity in perovskite oxide ferroelectrics (FEs) is crucial for devices based on these materials. Generally, FEs are wide-bandgap insulators possessing conductivity as small as $10^{-10}-10^{-12} \mathrm{~S} / \mathrm{cm}$. However, conductivity is known to increase to a semiconductor level of $10^{-8}-10^{-6} \mathrm{~S} / \mathrm{cm}$ and even to a metal-like one when heterovalent doping and/or oxygen vacancies are introduced in FE. In oxygen-deficient semiconducting FEs, the electronic $n$-type conductivity was experimentally determined (e.g., by direct Hall measurements) [1-7]. Concurrently, a $p$-type conductivity was hypothesized to explain minima in conductivity as a function of oxygen pressure during synthesis or measurements $[8,9]$. This hypothetic $p$ type of conductivity was not proven experimentally. However, it was speculated that motion of charged oxygen vacancies might lead to the $p$-type conductivity. The concept of oxygen vacancies as mobile charge carriers appeared convenient for simplistic explanations of electric-field-induced phenomena in FEs and is now increasingly popular [10-20].

The widely accepted basic constituents of the concept are as follows. First, the Kröger-Vink treatment of lattice defects is applied to perovskite oxide FEs [21]. Namely, it is assumed that when unintentional or intentional acceptor dopants are

This work was supported by the Czech Science Foundation (Grant No. 1909671S) and the European Structural and Investment Funds and the Ministry of Education, Youth and Sports of the Czech Republic through Programme "Research, Development and Education" (Project No. SOLID21 CZ.02.1.01/0.0/0.0/16_019/0000760). introduced in perovskite oxide, the oxide should contain doubly ionized oxygen vacancies $\mathrm{V}_{\mathrm{O}}^{+2}$, whose concentration is determined by that of the dopants. For instance, when such ions as $\mathrm{Fe}^{3+}, \mathrm{Al}^{3+}, \mathrm{K}^{+}, \mathrm{Na}^{+}, \mathrm{Mn}^{3+}$, etc, substitute at $\mathrm{Ti}^{4+}$ site in $A^{2+} \mathrm{Ti}^{4+} \mathrm{O}^{2-}{ }_{3}$, the vacancies $\mathrm{V}_{\mathrm{O}^{2+}}$ are expected to form in order to compensate acceptor-induced charge imbalance. Second, the charged vacancies $\mathrm{V}_{\mathrm{O}}^{2+}$ are considered as mobile, able to migrate under applied electric field and thus contribute to the electrical conductivity. The mobility of the vacancies is argued to be supported by the experimental observations of the hightemperature thermal diffusion of oxygen atoms [17, 22].

These basic constituents are strongly confronted by the modern understanding of physics of FEs. Primarily, perovskite oxide FEs cannot be thought of as ionic crystals, for which the Kröger-Vink approach is valid [23-28]. The presence of covalent bonding and the effective ion charges, which significantly differ from the nominal valences, were clearly demonstrated in FEs. Additionally, the presence of intrinsic free (in the conduction band) or nearly-free electrons was evidenced in FEs (see review Ref. 29 and references therein). Moreover, the concentration of the free electrons can be as large as $10^{17}$ $10^{20} \mathrm{~cm}^{-3}$ and ensure efficient compensation of charge imbalance [30]. Therefore, the Kröger-Vink reactions are far insufficient for establishing the formation, structure, and concentration of point defects and charge carriers in FEs.

The formation and structure of oxygen-vacancy-related defects is now better understood owing to the progress in the first-principles theory [31-36]. It was shown that in such FEs as $\mathrm{BaTiO}_{3}$ and $\mathrm{SrTiO}_{3}$, the oxygen vacancies, dopant ions, and related complexes can possess different charge and electronic (in-gap or band) states and, importantly, they can trap/release electrons.

Interestingly, it was also shown that even for the high temperatures above $1000 \mathrm{~K}$, at which oxygen vacancies and/or other point defects might move, the ionic conductivity due to such motion is several orders of magnitude smaller than the electronic conductivity and can be disregarded [37]. Ironically, there is a growing number of publications, where the electronic conductivity is disregarded instead.

So far, the only argument in favor of oxygen-vacancy-

M. Tyunina is with Microelectronics Research Unit, Faculty of Information Technology and Electrical Engineering, University of Oulu, P. O. Box 4500, FI-90014 Oulu, Finland (e-mail: marina.tjunina@oulu.fi) and Institute of Physics of the Czech Academy of Sciences, Na Slovance 2, 18221 Prague, Czech Republic (e-mail: tjunina@fzu.cz). 
determined conductivity may be the activation-type dependence of conductivity $\sigma$ on temperature: $\sigma \propto \exp (-$ $\left.E_{A} / k_{B} T\right)$, where $E_{A}$ and $k_{B}$ are the activation energy and the Boltzmann constant, respectively. The large activation energy $E_{A}=0.5-0.7 \mathrm{eV}$ exceeds that known for electron polarons in FEs [1-7] and, therefore, is assigned to hopping of oxygen vacancies $[13,14,17,20]$. In the present work, it is experimentally demonstrated that such processes as electrons' trapping, release, and excitation to the conduction band result in the energy $E_{A}$ up to $0.8 \mathrm{eV}$, which simply mimics the alleged motion of oxygen vacancies.

The experiments were as follows. Epitaxial stoichiometric and oxygen deficient $\mathrm{BaTiO}_{3}$ (BTO) films were studied as an archetypal example of FEs. The BTO films were grown on single-crystal (001) $\mathrm{SrTiO}_{3}$ (STO) substrates, which impose inplane biaxial compressive strain on BTO. The strain favors the formation and stabilization of oxygen vacancies in the $\mathrm{Ba}-\mathrm{O}$ atomic planes (but not in the Ti-O planes) parallel to the substrate surface [38-41]. Correspondingly, an out-of-plane motion of oxygen vacancies (i.e., from the Ba-O planes to the Ti-O planes) is prohibited in the films. The conductivity was measured along this peculiar out-of-plane direction using a small amplitude of the probing ac voltage, which dismissed an injection of excess carriers.

The obtained experimental results as well as charge transport in general, optical absorption, electro-coloration, and electrodegradation are discussed as originating from the electronic processes at the presence of immobile defects. The popular concept of mobile oxygen vacancies is inferred as invalid.

\section{EXPERIMENT}

Perovskite-structure BTO films were grown by pulsed laser deposition using the pressure of oxygen ambience $20 \mathrm{~Pa}$ for stoichiometric films and 0.1-2 Pa for oxygen-deficient ones. The 150-nm-thick (001)BTO films and bottom (001) $\mathrm{SRuO}_{3}$ (SRO) electrode layers were grown on (001) $\mathrm{STiO}_{3}$ (STO) substrates. Compared to the room-temperature unit cell of bulk BTO (volume $V_{0}$ ), the 0.1-2-Pa BTO films experience unit-cell expansion $\delta V=\left(V / V_{0}-1\right)$, which corroborates the presence of oxygen vacancies therein. Because of combined action of epitaxial strain and oxygen vacancies, the expansion $\delta V$ is not proportional to the vacancy concentration but characterizes it qualitatively. The detailed analyses of the films' composition, crystal structure, microstructure, and ferroelectric and optical properties can be found elsewhere [41,42].

For electrical characterization, the circular top Pt contact pads (area $0.2 \mathrm{~mm}^{2}$ ) were created by room-temperature vacuum pulsed laser deposition of $\mathrm{Pt}$ through a shadow mask. The impedance of the Pt/BTO/SRO capacitors was measured by a NOVOCONTROL Alpha-AN High Performance Frequency Analyzer using ac voltage with the amplitude of 1-10 mV and the frequency $f=\left(1-10^{6}\right) \mathrm{Hz}$. The voltage was applied and the response was measured along the out-of-plane direction of the capacitors. The impedance was analyzed using an equivalentcircuit model of a leaky parallel-plate capacitor [43-46].

The control of temperature was realized using a JANIS ST-
$100 \mathrm{He}$ flow cryostat equipped with a LakeShore 335 temperature controller. The temperature was swept at a rate of 3-5 K/min. The measurements were performed during (1) cooling from 300 to $80 \mathrm{~K}$, (2) heating from 80 to $710 \mathrm{~K}$, (3) cooling from 710 to $80 \mathrm{~K}$, and (4) consequent heating-cooling runs. The measurements were repeated after several weeks, during which the capacitors were kept at room temperature.

\section{RESULTS}

The conductivity $\sigma$ of the stoichiometric and oxygendeficient films was found to increase with temperature and frequency. The conductivity $\sigma_{0}$ measured at $f=1 \mathrm{~Hz}$ was used to follow the temperature evolution of the static conductivity. During the first heating to $710 \mathrm{~K}$, the magnitude and the temperature dependence of $\sigma_{0}$ in the oxygen-deficient film with $\delta V=2.7 \%$ resembled those in the stoichiometric film [Fig. 1 (a)]. Both these films exhibited very small $\sigma_{0}=10^{-13}-10^{-8} \mathrm{~S} / \mathrm{cm}$ at the temperatures $T<600 \mathrm{~K}$, that indicated the films' perfect insulating character. In the oxygen-deficient film with $\delta V=3.1$ $\%$, the low-temperature insulating state with $\sigma_{0}<10^{-8} \mathrm{~S} / \mathrm{cm}$ was found only at $T<200 \mathrm{~K}$, whereas a semiconductor-like increase of $\sigma_{0}$ at $T=200-450 \mathrm{~K}$ and a metal-like decrease of $\sigma_{0}$ at $T>$ $450 \mathrm{~K}$ were observed therein [Fig. 1(b)].
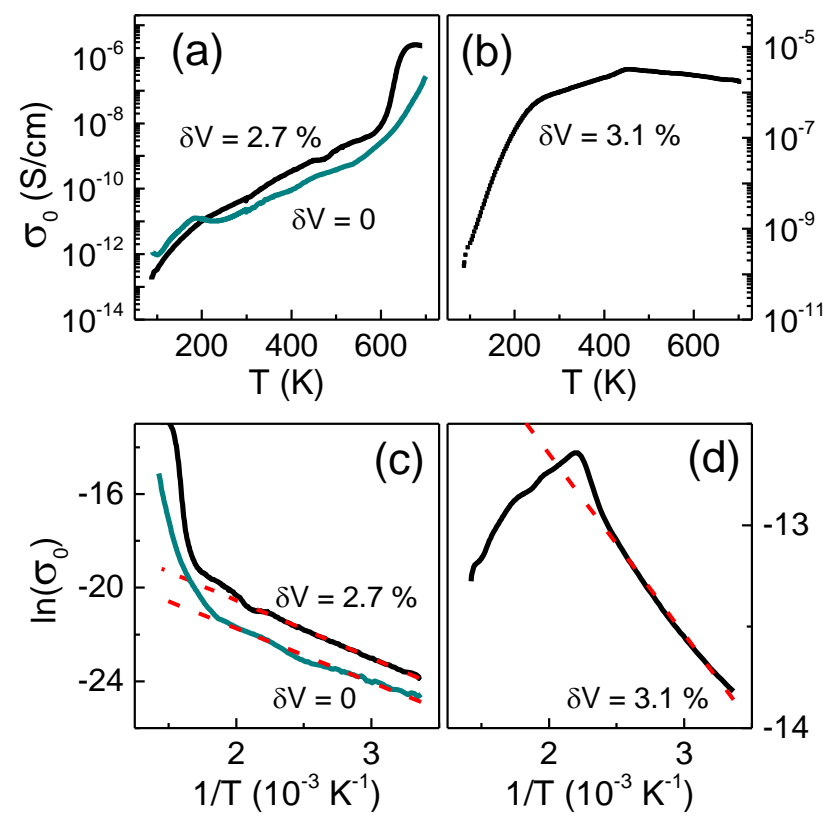

Fig. 1. (a, b) Conductivity $\sigma_{0}(f=1 \mathrm{~Hz})$ as a function of temperature during the first heating. (c, d) Activation character of the low-temperature conductivity.

In all films in the insulating state, the conductivity showed an activation type of behavior $\left[\sigma_{0} \propto \exp \left(-E_{A} / k_{B} T\right)\right]$ as evidenced by good linear fits to $\left.\left[\ln \left(\sigma_{0}\right) \propto 1 / T\right)\right]$ [Figs. 1(c, d)]. The activation energy $E_{A}$ was extracted from the fits and appeared to be $E_{A} \approx 0.2 \mathrm{eV}$ for $\delta V=0 \%$ and $\delta V=2.7 \%$, and $E_{A} \approx 0.08$ $\mathrm{eV}$ for $\delta V=3.1 \%$. The small magnitude of $E_{A}$ substantiated hopping of small electron polarons as responsible for the charge transport in the insulating state of the films [1-7].

To verify the polaronic hopping, the frequency dispersion of 
ac conductivity $\sigma(f)$ was analyzed [Fig. 2]. The power-law dependence $\left[\sigma=\sigma_{00}+A f^{s}\right]$ was applied, where the exponent $s$ $<1$ can disentangle between a temperature-independent quantum-mechanical transport and a temperature-dependent barrier hopping $[47,48]$. For the electron barrier hopping, the exponent was shown to be $s \approx 1$ at $T \rightarrow 0 \mathrm{~K}$ and decrease at higher temperatures. Here, the exponent $s$ was extracted from the fits to the power law. In the oxygen-deficient film with $\delta V$ $=2.7 \%$, the dc conductivity $\sigma_{00}$ increased, and the exponent $s$ decreased from $\sim 1$ to $\sim 0.8$ with increasing temperature from 80 to $450 \mathrm{~K}$ [Figs. 2(a, b)]. This behavior of $s$ corroborated the electronic hopping mechanism.
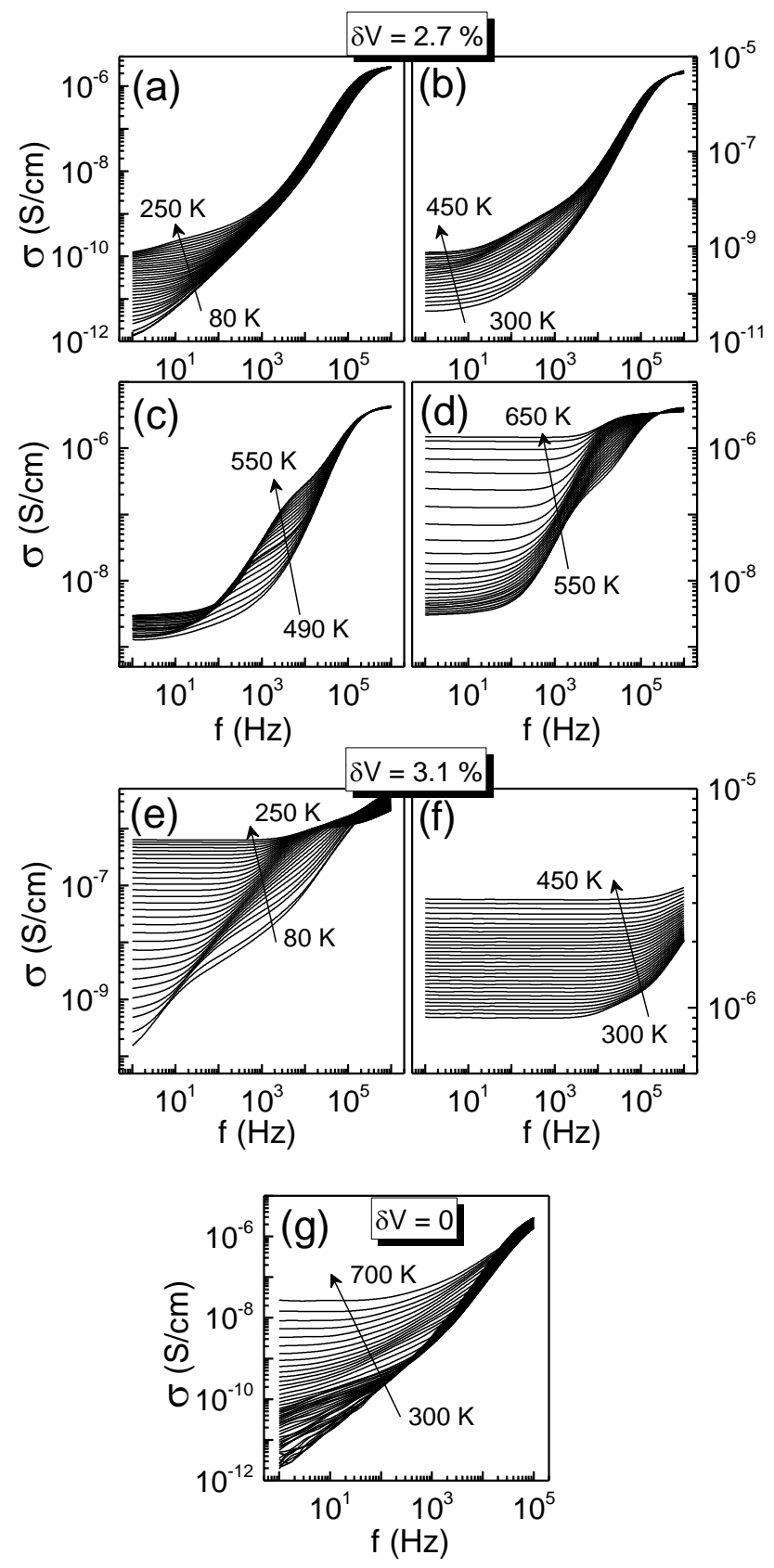

Fig. 2. Frequency dispersion of ac conductivity at different temperatures in the (a-d) oxygen-deficient and (g) stoichiometric films.
Closer inspections of the frequency dispersion at different temperatures [Fig. 2] revealed a coexistence of the hopping mechanism with another high-temperature process and a crossover to this high-temperature process, which was manifested by a complex dispersion $\sigma(f)$ [Figs. 2(c, d, e)]. The large and frequency-independent $\sigma \approx 10^{-6}-10^{-5} \mathrm{~S} / \mathrm{cm}$ indicated that this process is a conduction-band transport at $T>600 \mathrm{~K}$ in the film with $\delta V=2.7 \%$ [Fig. 2(d)]. For the stronger oxygen deficiency $(\delta V=3.1 \%)$, the polaronic hopping and band transport were found to coexist at lower temperatures [Fig. 2(e)], with the band transport dominating already at $T>250 \mathrm{~K}$ [Fig. 2(f)].

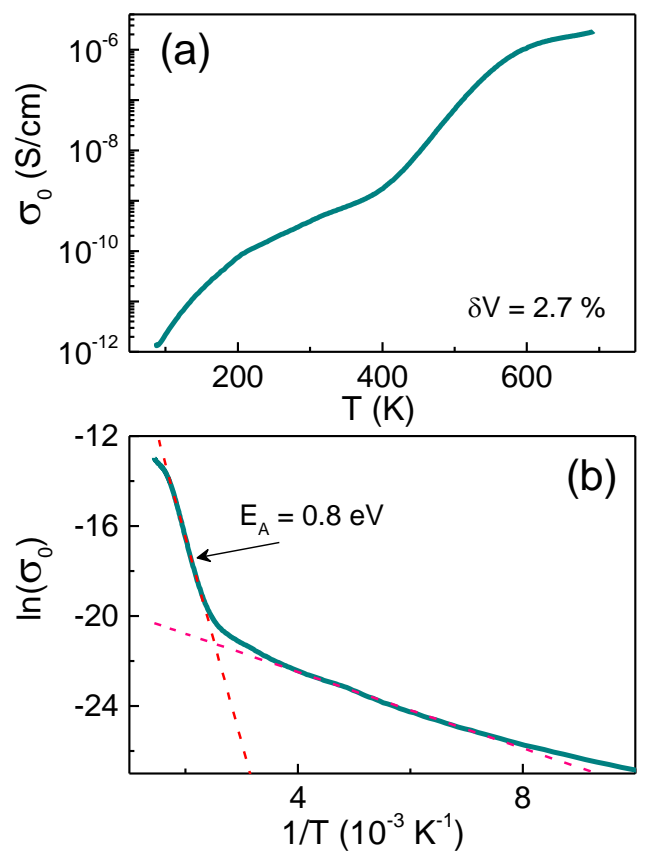

Fig. 3. (a) Conductivity $\sigma_{0}(f=1 \mathrm{~Hz})$ as a function of temperature and (b) activation character of $\sigma_{0}$ determined during cooling (after the first heating) in the film with $\delta V=2.7 \%$.

In the oxygen-deficient film with $\delta V=2.7 \%$, in addition to the polaronic hopping and band transport, a steep hightemperature increase of the conductivity by three orders of magnitude, to a metal-type level, was observed in the narrow temperature range of $T=600-650 \mathrm{~K}$ [Fig. 1(a)]. This behavior indicated a massive release of electrons from traps and electrons' excitation to the conduction band. During consequent thermal cycling, a dynamic equilibrium between the electronic release and trapping resulted in the band conductivity in a broader high-temperature region $T>370 \mathrm{~K}$ [Figs. 3(a) and 4(b)]. Importantly, this high-temperature large and frequencyindependent semiconductor-type band conductivity was accompanied by a good high-temperature activation fit to $\left.\left[\ln \left(\sigma_{0}\right) \propto 1 / T\right)\right]$ with the fitting energy $E_{A} \approx 0.8 \mathrm{eV}$ [Fig. 3(b)]. It should be stressed that the magnitude and frequency dispersion of the conductivity clearly endorsed the electronic band transport here. Therefore, an apparent large activation energy for the conductivity does not prove hopping of oxygen 
vacancies, in contrast to the widely spread belief.

During thermal cycling after the initial heating of the film with $\delta V=2.7 \%$, also the low-temperature insulating state and polaronic hopping were confirmed by the activation behavior $\left.\left[\ln \left(\sigma_{0}\right) \propto 1 / T\right)\right]$ with the energy $E_{A} \approx 0.07 \mathrm{eV}[$ Fig. 3(b)] and the hopping-type frequency dispersion $\sigma(f)$ at $T<300 \mathrm{~K}$ [Fig. 4(a)]. The hopping-to-band crossover was seen therein as well [Fig. 4(c)]. The initial behavior of conductivity [Figs. 1(a, c) and 2(ad)] was restored after several days of recovery at $300 \mathrm{~K}$.

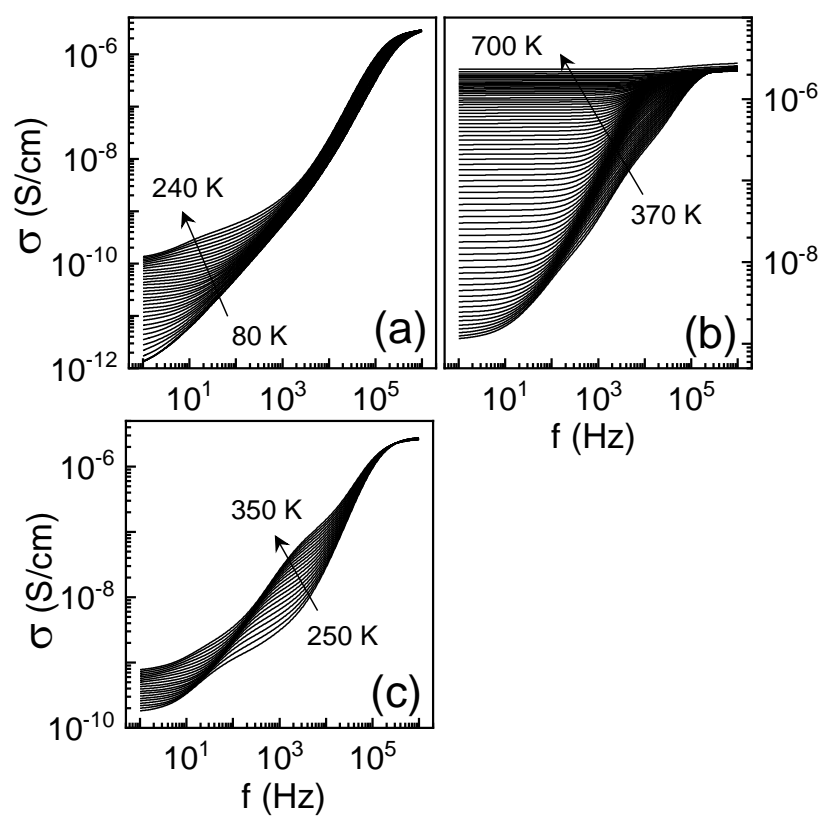

Fig. 4. Frequency dispersion of ac conductivity at different temperatures during cooling (after the first heating) in the film with $\delta V=2.7 \%$.

The high-temperature electronic release and excitation were detected in the stoichiometric film and in the strongly oxygen deficient film with $\delta V=3.7 \%$, too. Although the corresponding impact on the conductivity was less spectacular compared to that in the film with $\delta V=2.7 \%$.

The observed temperature- and frequency-dependent behavior of the small-signal conductivity imply the electronic transport in all BTO films, whereas oxygen vacancies are immobile. At low temperatures, the conductivity is due to the electron polaron barrier hopping. At high temperatures, the conductivity is of band character. The temperatures of the polaron-to-band crossover vary with the concentration of oxygen vacancies and thermal history. Importantly, the activation-type behavior with the large activation energy of 0.8 $\mathrm{eV}$ is found for the electronic band transport. The electronic processes, which determine the charge transport and electricfield-induced phenomena at the presence of immobile oxygen vacancies and/or other defects are discussed below.

\section{DISCUSSION}

According to the present understanding [31-36], in such FEs as BTO and STO, oxygen vacancies $\mathrm{V}_{\mathrm{O}}$ can exist as neutral $\left(\mathrm{V}_{\mathrm{O}}{ }^{0}\right)$, singly positive $\left(\mathrm{V}_{\mathrm{O}}^{+}\right)$, and/or doubly positive $\left(\mathrm{V}_{\mathrm{O}}^{+2}\right)$ ones.
The vacancy $\mathrm{V}_{\mathrm{O}}{ }^{+2}$ can trap one to two electrons in the form of small polarons. In such polarons, the electrons are localized on $\mathrm{Ti}$ atoms near the vacancy, that changes $\mathrm{Ti}^{4+}$ to $\mathrm{Ti}^{3+}$. Additionally, an isolated small polaron may occur as electron localized on a single Ti atom (self-trapped electron polaron). The lowest formation energy is found for the doubly charged vacancies $\mathrm{V}_{\mathrm{O}}{ }^{+2}$ and the complexes of $\mathrm{V}_{\mathrm{O}}{ }^{+2}$ and one polaron, or $\left(\mathrm{V}_{\mathrm{O}}{ }^{+2}-\mathrm{Ti}^{3+}\right)^{+}$.

In terms of electronic band structure [Fig. 5(a)], the neutral $\mathrm{V}_{\mathrm{O}}{ }^{0}$ gives the occupied in-gap state $\sim 0.1-0.2 \mathrm{eV}$ below the conduction band. For the singly charged $\mathrm{V}_{\mathrm{O}}^{+}$vacancy, the occupied state is $\sim 0.5 \mathrm{eV}$ below the conduction band. The doubly charged $\mathrm{V}_{\mathrm{O}}{ }^{+2}$ vacancy has no in-gap states but contributes to the conduction band. The complex of $\mathrm{V}_{\mathrm{O}}{ }^{+2}$ with one polaron, i.e. $\left(\mathrm{V}_{\mathrm{O}}^{+2}-\mathrm{Ti}^{3+}\right)^{+}$, is the most stable configuration. Based on the experiments $[49,50]$, the $\left(\mathrm{V}_{\mathrm{O}}{ }^{+2}-\mathrm{Ti}^{3+}\right)^{+}$-related ingap occupied states are $\sim 0.6 \mathrm{eV}$ above the valence band.

It is worth noting that the presence of acceptor dopants such as $\mathrm{Fe}$ does not necessarily promote the formation of the $\mathrm{V}_{\mathrm{O}}{ }^{+2}$ vacancies. The presence of iron $\mathrm{Fe}^{4+}, \mathrm{Fe}^{3+}$, and $\mathrm{Fe}^{2+}$, neutral oxygen vacancies $\mathrm{V}_{\mathrm{O}}{ }^{0}$, and $\mathrm{Fe}^{3+}-\mathrm{V}_{\mathrm{O}}$ complexes was detected instead $[33,34]$. The corresponding occupied in-gap states were found at $\sim 0.5 \mathrm{eV}$ below the conduction band and $\sim 0.1 \mathrm{eV}$ above the valence band.

In an undoped FE BTO, the vacancies $\mathrm{V}_{\mathrm{O}}{ }^{0}, \mathrm{~V}_{\mathrm{O}}{ }^{+}$, and $\mathrm{V}_{\mathrm{O}}{ }^{+2}$ and the $\left(\mathrm{V}_{\mathrm{O}}{ }^{+2}-\mathrm{Ti}^{3+}\right)^{+}$complexes can form [Fig. 5(a)]. The most favorable states are $\mathrm{V}_{\mathrm{O}}{ }^{+2}$ and $\left(\mathrm{V}_{\mathrm{O}}{ }^{+2}-\mathrm{Ti}^{3+}\right)^{+}$. These states are considered here for the sake of simplicity. (a)

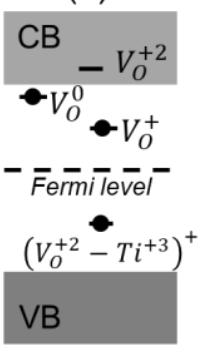

(b)

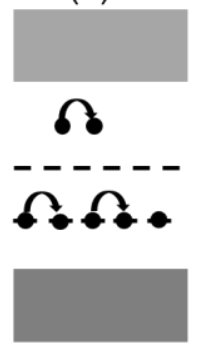

(c)

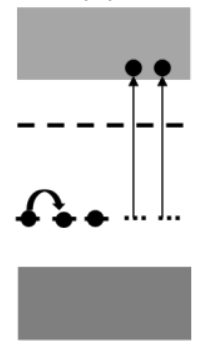

(d)

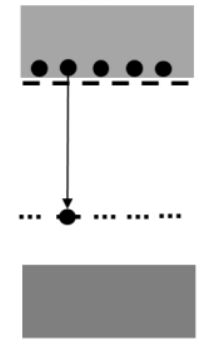

Fig. 5. Schematic band diagrams of FE showing (a) oxygen-vacancy related electronic states and (b-c) thermally activated electronic excitations.

At the absence of external electron injection, the charge transport at different temperatures is schematically illustrated in Figs. 5(b-d). At low temperatures, the charge transport is realized by hopping of small electron polarons, which are selftrapped or associated with the $\left(\mathrm{V}_{\mathrm{O}}{ }^{+2}-\mathrm{Ti}^{3+}\right)^{+}$complexes [Fig. 5(b)]. With increasing temperature, the electrons are excited thermally and released from the $\left(\mathrm{V}_{\mathrm{O}}{ }^{+2}-\mathrm{Ti}^{3+}\right)^{+}$traps [Fig. 5(c)]. The $\left(\mathrm{V}_{\mathrm{O}}{ }^{+2}-\mathrm{Ti}^{3+}\right)^{+}$complexes turn into the $\mathrm{V}_{\mathrm{O}}{ }^{+2}$ states, which mimics an arrival of the $\mathrm{V}_{\mathrm{O}^{+2}}$ vacancies, although the vacancies are immobile. Concurrently, the concentration of polarons decreases and, hence, the conductivity can decrease, which imitates a $p$-type contribution to the charge transport. Next, the conduction band is being filled with the released electrons and, correspondingly, the Fermi level is rising. These changes lead 
to a coexistence of the polaronic and conduction-band mechanisms of conductivity. Finally, at high temperatures, the large concentration of the conduction-band electrons and the Fermi level at the bottom of the conduction band can be achieved, which determines semiconductor- or metal-type conductivity [Fig. 5(d)].

For the thermally-activated electronic processes in Figs. 5(bc), the concentration of the mobile charge carriers is proportional to $\exp \left(-E_{A} / k_{B} T\right)$, where the activation energy $E_{A}$ takes values between the polaron binding energy of $\sim 0.2-0.3 \mathrm{eV}$ and a half of the bandgap, i.e. $~ 1.6-1.7 \mathrm{eV}$ [51]. The large energy $E_{A}$ for the electronic concentration and, hence, conductivity imitates the energy, which was erroneously interpreted before as that for hopping of oxygen vacancies.

Thus, the electronic release from the traps and the electrons' re-trapping give a feeling of the arrival and departure of the $\mathrm{V}_{\mathrm{O}}{ }^{+2}$ vacancies, $p$-type conductivity, and thermally activated motion of the $\mathrm{V}_{\mathrm{O}}^{+2}$ vacancies, whereas the vacancies are immobile. Importantly, as seen from the schematics in Figs. 5(b-d), not only oxygen vacancies, but also other immobile defects producing in-gap states and electron traps, can similarly affect the charge transport. (a)

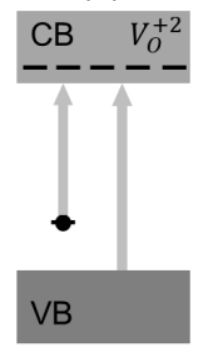

(b)

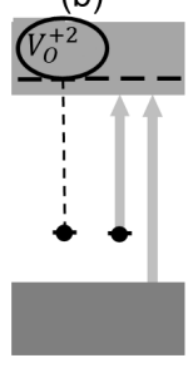

(c)

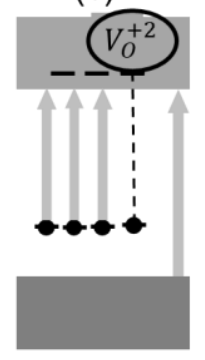

(d)

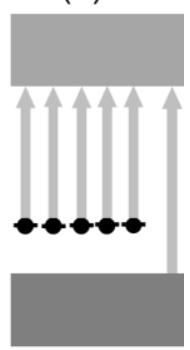

Fig. 6. Schematics of the band-to-band and trap-to-band optical transitions at the (a) absence and (b-d) presence of the injected electrons. The electron trapping by the $\mathrm{V}_{\mathrm{O}}{ }^{+2}$ vacancy into the $\left(\mathrm{V}_{\mathrm{O}}{ }^{+2}-\mathrm{Ti}^{3+}\right)^{+}$state is also shown.

In contrast to the small-signal conductivity at the absence of electron injection, applying external dc electric field to the electroded FE sample leads to injection of excess electrons from the electrode (cathode) into FE. The injected electrons can be trapped/released by immobile defects and influence the conductivity as in Fig. 5(c). When a certain large enough concentration of the injected electrons is achieved, the insulating state of FE turns into a semiconducting one [Fig. 5(d)]. Notably, because of different characteristic times for the electronic release and trapping, the dc conductivity can exhibit hysteresis upon sweeping the $\mathrm{dc}$ electric field (resistive switching).

Besides the conductivity, the concept of mobile oxygen vacancies is believed to be mandatory to explain electrocoloration and de degradation of FEs in the first place [10-12]. As shown next, purely electronic processes can explain these phenomena.

Under applied dc electric field, the injected electrons can be responsible for an increase of the optical absorption in the visible spectral range, or the so-called coloration effect [Fig. 6]. For instance, in the presence of the most favorable states $\mathrm{V}_{\mathrm{O}^{+2}}$ and $\left(\mathrm{V}_{\mathrm{O}}{ }^{+2}-\mathrm{Ti}^{3+}\right)^{+}$, the optical absorption is determined by the electronic excitations from the valence band to the conduction band and from the $\left(\left(\mathrm{V}_{\mathrm{O}}{ }^{+2}-\mathrm{Ti}^{3+}\right)^{+}\right.$states to the conduction band [Fig. 6(a)]. The $\mathrm{V}_{\mathrm{O}}{ }^{+2}$ states are in the conduction band and, therefore, do not contribute to the absorption. The injected electrons are trapped by the $\mathrm{V}_{\mathrm{O}}{ }^{+2}$ vacancies, which produces the occupied in-gap $\left(\mathrm{V}_{\mathrm{O}}{ }^{+2}-\mathrm{Ti}^{3+}\right)^{+}$states. The filling of the traps and generation of additional occupied in-gap states lead to an additional absorption [Fig. 6(b)]. With increasing concentration of the injected electrons, the absorption increases [Fig. 6(c)]. At a very high concentration of the injection-induced occupied ingap states, the absorption edge is dominated by these states and shifts to lower photon energies [Fig. 6(d)]. In addition to (or instead of) oxygen vacancies, also other in-gap electronic traps (e.g., due to dopants) can be filled with the injected electrons and, thus, contribute to electro-coloration.

The excess electrons, which are injected from the cathode, can fill the conduction band of the initially insulating FE [Fig. 7(a)]. In the near-cathode region, the injection-induced semiconductor FE adjoins the insulating FE. This contact can be treated as a semiconductor homojunction with the contact potential $\Delta V_{C}$ [Figs. 7(a-b)] [51]. The formed junction is forward-biased by the external dc field, which lowers the contact potential [Figs. 7(c-d)] and enables motion of the injected electrons further away from the cathode. Correspondingly, the semiconductor-insulator boundary moves from the cathode towards the anode when more and more electrons are injected with time during the dc biasing [Figs. 8(ac)]. The injected electrons gradually reach the sample regions at progressively longer distances from the cathode that leads to the apparent propagation of the coloration boundary.

\section{(b)}

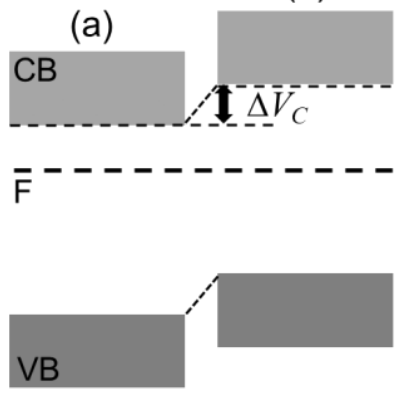

(c)
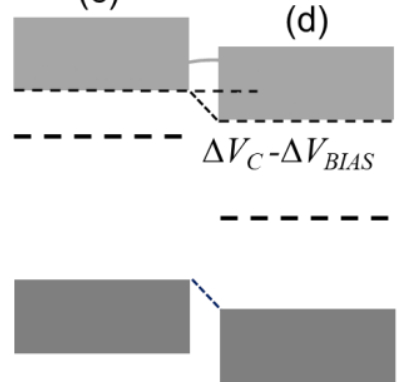

Fig. 7. Schematic band diagrams of contacts between $(a, c)$ semiconducting FE and (b, d) insulating FE at the (a-b) absence and (c-d) presence of external bias.

The injection and motion of electrons from the cathode can explain dc degradation as well. The initial thickness of the insulating FE is $d$ and the strength of the applied dc electric field is $E_{D C}=\Delta V_{B I A S} / d$, correspondingly [Fig. 8(a)]. Upon continuous $\mathrm{dc}$ biasing and electronic injection from the cathode, the semiconductor-insulator boundary is formed and moves towards the anode [Figs. 8(b-c)], whereas the current is still limited by the presence of the insulating FE. However, the effective thickness of the insulator decreases with time, which 
results in an increase of the field strength across the insulating fraction to $E_{D C}=\Delta V_{B I A S} /(d-\Delta d)$. When the effective thickness reaches a small critical value of $d^{*}$, the large electrical field $E_{D C}$ $=\Delta V_{\text {BIAS }} / d^{*}$ causes breakdown [Fig. 8(d)]. Remarkably, the injection and motion of electrons does not require presence of oxygen vacancies and/or other defects at all. The dc degradation can therefore occur in a perfect undoped crystal when injection of electrons is allowed. Likewise, the degradation time can be significantly prolonged by raising the FE-cathode barrier for electron injection.
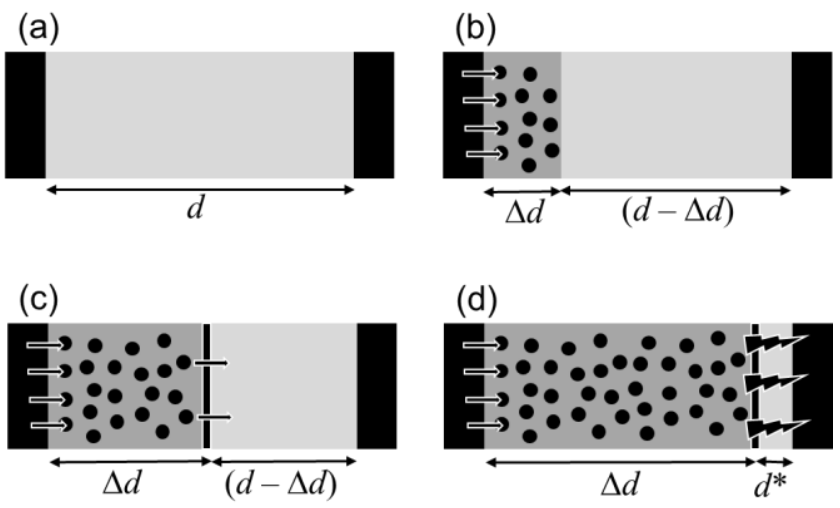

Fig. 8. Schematics of FE degradation under applied dc electric field in FE: (a) initial state, (b) electronic injection from the cathode, (c) motion of the semiconductor-insulator boundary, (d) breakdown.

As demonstrated here, such electronic processes as hopping, trapping by immobile defects, release (or de-trapping), excitation to the conduction band, and electric-field induced injection and motion from the cathode explain all phenomena which were previously vaguely attributed to mobile oxygen vacancies. The hypothesis of mobile oxygen vacancies is likely invalid. However, the electronic structure and properties of FEs can be significantly affected by immobile oxygen vacancies and/or other defects, which demand more scrutiny.

\section{CONCLUSIONS}

The small-signal conductivity is investigated as a function of temperature and frequency in oxygen-deficient and reference stoichiometric barium titanate films. The observations evidence pure electronic processes, whereas oxygen vacancies are stabilized by epitaxial strain. The electronic processes are shown to mimic motion of oxygen vacancies, which are immobile.

The electronic hopping, trapping/release by defects, excitation to the conduction band, and field-induced injection and motion from the cathode are demonstrated to explain all features of the electrical conductivity, electro-coloration, and degradation, which were previously hypothetically ascribed to mobile oxygen vacancies.

The concept of mobile oxygen vacancies is inferred as misleading for understanding electrical and optical behavior of perovskite oxide ferroelectrics.

\section{ACKNOWLEDGMENT}

The author is grateful to T. Kocourek, J. Peräntie, and M. Savinov for preparation and characterization of the samples.

\section{REFERENCES}

[1] P. Gerthsen, K. H. Hardtl, A. Csillag, "Mobility Determinations from Weight Measurements in Solid Solutions of $(\mathrm{Ba}, \mathrm{Sr}) \mathrm{TiO}_{3}$," Phys. Stat. Sol. (a), vol. 13, pp. 127-133, Sept. 1972.

[2] H. Yamada and G. R. Miller, "Point Defects in Reduced Strontium Titanate," J. Solid State Chem., vol. 6, pp. 169-177, Jan. 1973.

[3] H. Ihrig and D. Hennings, "Electrical transport properties of $n$-type BaTiO 3 ," Phys. Rev. B, vol. 17, pp. 4593-4599, June 1978.

[4] T. Kolodiazhnyi, A. Petric, aM. Niewczas, C. Bridges, A. Safa-Sefat, J. E. Greedan, "Thermoelectric power, Hall effect, and mobility of $n$-type BaTiO $_{3}$," Phys. Rev. B, vol. 68, pp. 085205-1-5, Aug. 2003.

[5] T. Kolodiazhnyi, "Insulator-metal transition and anomalous sign reversal of the dominant charge carriers in perovskite $\mathrm{BaTiO}_{3-\delta}$, Phys. Rev. B, vol. 78, 045107-1-5, July 2008.

[6] J. P. Boyeaux, F.M. Michel-Calendini, "Small polaron interpretation of $\mathrm{BaTiO}_{3}$ transport properties from drift mobility measurements," J. Phys. C: Solid State Phys., vol. 12, pp. 545-556, July 1979.

[7] E. Iguchi, N. Kubota, T. Nakamori, N. Yamamoto, K. J. Lee, "Polaronic conduction in $n$-type $\mathrm{BaTiO}_{3}$ doped with $\mathrm{La}_{2} \mathrm{O}_{3}$ or $\mathrm{Gd}_{2} \mathrm{O}_{3}$," Phys. Rev. $B$, vol. 43, pp. 8646-8649, April 1991.

[8] S. A. Long and R. N. Blumenthal, "Ti-Rich Nonstoichiometric $\mathrm{BaTiO}_{3}$ : I, High Temperature Electrical Conductivity Measurements," J. Am. Ceram. Soc., vol. 54, pp. 515-519, Oct. 1971.

[9] N. H. Chan, R. K. Sharma, D. M. Smyth, "Nonstoichiometry in Undoped $\mathrm{BaTiO}_{3}$," J. Am. Ceram. Soc., vol. 64, pp. 556-562, Sept. 1981.

[10] R. Waser, T. Baiatu, K.-H. Hardtl, "dc Electrical Degradation of Perovskite-Type Titanates: I, Ceramics," J. Am. Ceram. Soc., vol. 73, pp. 1645-1653, June 1990.

[11] R. Waser, T. Baiatu, K. H. Hardtl, "dc Electrical Degradation of Perovskite-Type Titanates: II, Single Crystals," J. Am. Ceram. Soc., vol. 73, pp. 1654-1662, June 1990.

[12] T. Baiatu, R. Waser, K. H. Hardtl, "dc Electrical Degradation of Perovskite-Type Titanates: III, A Model of the Mechanism," J. Am. Ceram. Soc., vol. 73, pp. 1663-1673, June 1990.

[13] C. Ang, Z. Yu, L. E. Cross, "Oxygen-vacancy-related low-frequency dielectric relaxation and electrical conduction in $\mathrm{Bi}_{\mathrm{S}} \mathrm{SrTiO}_{3}$, , Phys. Rev. $B$, vol. 62, pp. 228-236, July 2000.

[14] B. S. Kang and S. K. Choi, C. H. Park, "Diffuse dielectric anomaly in perovskite-type ferroelectric oxides in the temperature range of 400700'," J. Appl. Phys., vol. 94, pp. 1904-1911, July 2003.

[15] R. Meyer, R. Liedtke, R. Waser, Oxygen vacancy migration and timedependent leakage current behavior of $\mathrm{Ba}_{0.3} \mathrm{Sr}_{0.7} \mathrm{TiO}_{3}$ thin films, Appl. Phys. Lett., vol. 86, pp. 112904-1-3, March 2005.

[16] A. G. Boni, I. Pintilie, L. Pintilie, D. Preziosi, H. Deniz, M. Alexe, "Electronic transport in (La,Sr) $\mathrm{MnO}_{3}$-ferroelectric-(La, $\left.\mathrm{Sr}\right) \mathrm{MnO}_{3}$ epitaxial structures," J. Appl. Phys., vol. 113, pp. 2241031-10, June 2013.

[17] R. A. Maier, C. A. Randall, "Low Temperature Ionic Conductivity of an Acceptor-Doped Perovskite: II. Impedance of Single-Crystal $\mathrm{BaTiO}_{3}$," J. Am. Ceram. Soc., vol. 99, pp. 3360-3366, June 2016.

[18] Y. Heo, D. Kan, Y. Shimakawa, J. Seidel, "Resistive switching properties of epitaxial $\mathrm{BaTiO}_{3-\delta}$ thin films tuned by after-growth oxygen cooling pressure," Phys. Chem. Chem. Phys., vol. 18, 197-204, Nov. 2016.

[19] M. Li, J. Zhou, X. Jing, M. Zeng, S. Wu, J. Gao, Z. Zhang, X. Gao, X. Lu, J.-M. Liu, M. Alexe, "Controlling Resistance Switching Polarities of Epitaxial $\mathrm{BaTiO}_{3}$ Films by Mediation of Ferroelectricity and Oxygen Vacancies," Adv. Electron. Mater., pp. 1500069-1-8, April 2015.

[20] N. Du, N. Manjunath, Y. Li, S. Menzel, E. Linn, R. Waser, T. You, D. Bürger, I. Skorupa, D. Walczyk, C. Walczyk, O. G. Schmidt, H. Schmidt, "Field-Driven Hopping Transport of Oxygen Vacancies in Memristive Oxide Switches with Interface-Mediated Resistive Switching," Phys. Rev. Appl., vol. 10, pp. 054025-1-8, Nov. 2018. 
TUFFC-09969-2020

[21] F. A. Kröger and H. J. Vink, "Relations Between Concentrations of Imperfections in Crystalline Solids," in Solid State Physics, vol. 3, F. Seitz and D. Turnbull, Academic Press, New York, 1956, pp. 307-435.

[22] M. Kessel, R. A. De Souza, and M. Martin, "Oxygen Diffusion in Single Crystal Barium Titanate," Phys. Chem. Chem. Phys., vol. 17, pp. 1258712597, April 2015.

[23] R. E. Cohen, "Origin of ferroelectricity in oxide ferroelectrics," Nature, vol. 358, pp. 136-138, July 1992.

[24] R. D. King-Smith, D. Vanderbilt, "First-principles investigation of ferroelectricity in perovskite compounds," Phys. Rev. B, vol. 49, pp. 5828-5844, March 1994.

[25] M. Posternak, R. Resta, A. Baldereschi, "Role of covalent bonding in the polarization of perovskite oxides: The case of $\mathrm{KNbO}_{3}$," Phys. Rev. B, vol. 50, 8911-8914, Sept. 1994.

[26] D. Vanderbilt, First-principles based modelling of ferroelectrics, Curr. Opin. Solid State Mater. Sci., vol. 2, pp. 701-705, Jan. 1997.

[27] Ph. Ghosez, J.-P. Michenaud, X. Gonze, "Dynamical atomic charges: The case of $\mathrm{ABO}_{3}$ compounds," Phys. Rev. B, vol. 58, pp. 6224-6240, Sept. 1998.

[28] R. E. Cohen, "Theory of ferroelectrics: a vision for the next decade and beyond," J. Phys. Chem. Solids, vol. 61, pp. 139-146, June (2000).

[29] Y. Watanabe, "Intrinsic Free Electrons/Holes at Polarization Discontinuities and their Implications for Basics of Ferroelectricity and its Origin," Solid State Phenom., vol. 189, pp. 57-93, June 2012.

[30] 3L. Pintilie, M. Alexe, "Metal-ferroelectric-metal heterostructures with Schottky contacts. I. Influence of the ferroelectric properties," J. Appl. Phys., vol. 98, pp. 124103-1-8, Dec. 2005.

[31] D. Gryaznov, E. Blokhin, A. Sorokine, E. A. Kotomin, R. A. Evarestov, A. Bussmann-Holder, J. Maier, "A Comparative $\mathrm{Ab}$ Initio Thermodynamic Study of Oxygen Vacancies in $\mathrm{ZnO}$ and $\mathrm{SrTiO}_{3}$ : Emphasis on Phonon Contribution," J. Phys. Chem. C, vol. 117, pp. 13776-1374, June 2013.

[32] R. Evarestov, E. Blokhin, D. Gryaznov, E. A. Kotomin, R. Merkle, J. Maier, "Jahn-Teller effect in the phonon properties of defective $\mathrm{SrTiO}_{3}$ from first principles," Phys. Rev. B, vol. 85, pp. 174303-1-5, May 2012.

[33] E. Blokhin, E. Kotomin, A. Kuzmin, J. Purans, R. Evarestov, J. Maier, "Theoretical modeling of the complexes of iron impurities and oxygen vacancies in $\mathrm{SrTiO}_{3}$," Appl. Phys. Lett., vol. 102, pp. 112913-1-4, March 2013.

[34] J. Kubacki, D. Kajewski, J. Goraus, K. Szot, A. Koehl, Ch. Lenser, R. Dittmann, J. Szade, "Impact of Fe doping on the electronic structure of $\mathrm{SrTiO}_{3}$ thin films determined by resonant photoemission," J. Chem. Phys., vol. 148, pp. 154702-1-8, April 2018.

[35] A. Janotti, J. B. Varley, M. Choi, C. G. Van de Walle, "Vacancies and small polarons in $\mathrm{SrTiO}_{3}$," Phys. Rev. B, vol. 90, pp. 085202-1-6, Aug. 2014.

[36] V. E. Alexandrov, E. A. Kotomin, J. Maier, and R. A. Evarestov, Firstprinciples study of bulk and surface oxygen vacancies in $\mathrm{SrTiO} 3$ crystal, Eur. Phys. J. B 72, vol. 53, pp. 53-57, Oct. 2009.

[37] P. Erhart, K. Albe, "Modeling the electrical conductivity in $\mathrm{BaTiO}_{3}$ on the basis of first-principles calculations," J. Appl. Phys., vol. 104, pp. 044315-1-8, Aug. 2008.

[38] A. Herklotz, D. Lee, E.-J. Guo, T. L. Meyer, J. R. Petrie, H. N. Lee, "Strain coupling of oxygen nonstoichiometry in perovskite thin films," J. Phys.: Condens. Matter, vol. 29, pp. 493001-1-16, Nov. 2017.

[39] U. Aschauer, N. A. Spaldin, "Interplay between strain, defect charge state, and functionality in complex oxides," Appl. Phys. Lett., vol. 109, pp. 031901-1-4, July 2016.

[40] J. Xi, H. Xu, Y. Zhang, W. J. Weber, "Strain effects on oxygen vacancy energetics in $\mathrm{KTaO}_{3}$," Phys. Chem. Chem. Phys., vol. 19, pp. 6264-6273, Feb. 2017.

[41] M. Tyunina, D. Chvostova, A. Dejneka, "Optical revelation of defects in epitaxial barium titanate films," Phys. Chem. Chem. Phys., vol. 21, pp. 7874-7478, March 2019.

[42] M. Tyunina, O. Pacherova, J. Peräntie, M. Savinov, M. Jelinek, H. Jantunen, A. Dejneka, "Perovskite ferroelectric tuned by thermal strain," Sci. Rep., vol. 9, pp. 3677-1-7, March 2019.

[43] M. Tyunina, J. Levoska, "Application of the interface capacitance model to thin-film relaxors and ferroelectrics," Appl. Phys. Lett., vol. 88, pp. 262904-1-3, June 2006.

[44] M. Tyunina, "Size effects and dielectric behaviour in ferroelectric heterostructures", J. Phys.: Condens. Matter, vol. 18, pp. 5725-5738, June 2006

[45] M. Tyunina, M. Plekh, M. Antonova, A. Kalvane, "Ferroelectric transitions in epitaxial $\mathrm{Pb}_{0.5} \mathrm{Sr}_{0.5} \mathrm{TiO}_{3}$ films studied by dielectric analysis," Phys. Rev. B, vol. 84, pp. 224105-1-10, Dec. 2011.

[46] M. Tyunina, B. Malic, M. Plekh, M. Kosec, "Dielectric Response of $\mathrm{BaTiO}_{3}$ Thin Film with Grain Size at Nanometer Scale," J. Am. Ceram. Soc., vol. 95, pp. 1333-1338, April 2012.

[47] A. Ghosh, "Frequency-dependent conductivity in bismuth-vanadate glassy semiconductors," Phys. Rev. B, vol. 41, pp. 1479-1488, Jan. 1990.

[48] A. Ghosh, "Correlated-barrier hopping in semiconducting tellurium molybdate glass," Phys. Rev. B, vol. 45, pp. 11318-11320, May 1992.

[49] C. N. Berglund, H. J. Braun, "Optical Absorption in Single-Domain Ferroelectric Barium Titanate," Phys. Rev., vol. 164, pp. 790-799, Dec. 1967.

[50] R. Scharfschwerdt, A. Mazur, O. F. Schirmer, H. Hesse, S. Mendricks, "Oxygen vacancies in $\mathrm{BaTiO}_{3}$," Phys. Rev. B, vol. 54, pp. 15284-15290, Dec. 1996

[51] C. M. Wolfe, N. Holonyak Jr., G. E. Stillman, Physical Properties of Semiconductors. Prentice Hall, 1989.

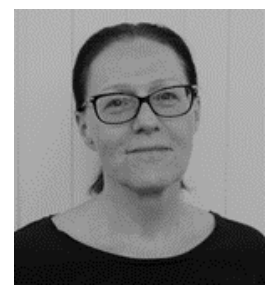

M. Tyunina (Tjunina) is a Researcher at University of Oulu and Institute of Physics, Prague. She obtained degrees of M.S. (physics, condensed matter physics) from University of Latvia, Ph.D. (condensed matter physics) from Moscow Engineering Physics Institute MEPhI, and D.Tech. (electronics) and Docent (materials physics) from University of Oulu. She worked in microelectronics industry for nearly a decade, as a Researcher at University of Latvia, and held invited Researcher/Professor positions in Sweden, Finland, Austria, and France. Her fields of expertise range from technology of semiconductor integrated circuits, laser-matter interactions, and pulsed laser deposition to physics of perovskite oxide relaxors, ferroelectrics, and related materials. Her recent research was focused on effects of epitaxy for tailoring electrical, optical, and mechanical response functions in perovskite oxides. 